\title{
The effect of protein dielectric coefficient on the ionic selectivity of a calcium channel
}

Douglas Henderson

Dezso Boda

Monika Valisko

Bob Eisenberg

Wolfgang Nonner

See next page for additional authors

Follow this and additional works at: https://scholarsarchive.byu.edu/facpub

Part of the Biochemistry Commons, and the Chemistry Commons

\section{Original Publication Citation}

Boda, Dezso, Monika Valisko, Bob Eisenberg, Wolfgang Nonner, Douglas Henderson, and Dirk Gillespie."The effect of protein dielectric coefficient on the ionic selectivity of a calcium channel." The Journal of Chemical Physics 125 (26).

\section{BYU ScholarsArchive Citation}

Henderson, Douglas; Boda, Dezso; Valisko, Monika; Eisenberg, Bob; Nonner, Wolfgang; and Gillespie, Dirk, "The effect of protein dielectric coefficient on the ionic selectivity of a calcium channel" (2006). Faculty Publications. 303.

https://scholarsarchive.byu.edu/facpub/303

This Peer-Reviewed Article is brought to you for free and open access by BYU ScholarsArchive. It has been accepted for inclusion in Faculty Publications by an authorized administrator of BYU ScholarsArchive. For more information, please contact ellen_amatangelo@byu.edu. 


\section{Authors}

Douglas Henderson, Dezso Boda, Monika Valisko, Bob Eisenberg, Wolfgang Nonner, and Dirk Gillespie 


\title{
The effect of protein dielectric coefficient on the ionic selectivity of a calcium channel
}

\author{
Dezső Boda ${ }^{a)}$ \\ Department of Molecular Biophysics and Physiology, Rush University Medical Center, Chicago, \\ Illinois 60612 and Department of Physical Chemistry, Pannon University, P.O. Box 158, \\ H-8201, Veszprém, Hungary \\ Mónika Valiskó \\ Department of Physical Chemistry, Pannon University, P.O. Box 158, H-8201, Veszprém, Hungary \\ Bob Eisenberg \\ Department of Molecular Biophysics and Physiology, Rush University Medical Center, Chicago, \\ Illinois 60612 \\ Wolfgang Nonner \\ Department of Physiology and Biophysics, University of Miami School of Medicine, Miami, Florida 33101 \\ Douglas Henderson \\ Department of Chemistry and Biochemistry, Brigham Young University, Provo, Utah 84602 \\ Dirk Gillespie \\ Department of Molecular Biophysics and Physiology, Rush University Medical Center, Chicago, \\ Illinois 60612
}

(Received 21 April 2006; accepted 17 May 2006; published online 18 July 2006)

Calcium-selective ion channels are known to have carboxylate-rich selectivity filters, a common motif that is primarily responsible for their high $\mathrm{Ca}^{2+}$ affinity. Different $\mathrm{Ca}^{2+}$ affinities ranging from micromolar (the $L$-type $\mathrm{Ca}$ channel) to millimolar (the ryanodine receptor channel) are closely related to the different physiological functions of these channels. To understand the physical mechanism for this range of affinities given similar amino acids in their selectivity filters, we use grand canonical Monte Carlo simulations to assess the binding of monovalent and divalent ions in the selectivity filter of a model Ca channel. We use a reduced model where the electolyte is modeled by hard-sphere ions embedded in a continuum dielectric solvent, while the interior of protein surrounding the channel is allowed to have a dielectric coefficient different from that of the electrolyte. The induced charges that appear on the protein/lumen interface are calculated by the induced charge computation method [Boda et al., Phys. Rev. E 69, 046702 (2004)]. It is shown that decreasing the dielectric coefficient of the protein attracts more cations into the pore because the protein's carboxyl groups induce negative charges on the dielectric boundary. As the density of the hard-sphere ions increases in the filter, $\mathrm{Ca}^{2+}$ is absorbed into the filter with higher probability than $\mathrm{Na}^{+}$because $\mathrm{Ca}^{2+}$ provides twice the charge to neutralize the negative charge of the pore (both structural carboxylate oxygens and induced charges) than $\mathrm{Na}^{+}$while occupying about the same space (the charge/space competition mechanism). As a result, $\mathrm{Ca}^{2+}$ affinity is improved an order of magnitude by decreasing the protein dielectric coefficient from 80 to 5 . Our results indicate that adjusting the dielectric properties of the protein surrounding the permeation pathway is a possible way for evolution to regulate the $\mathrm{Ca}^{2+}$ affinity of the common four-carboxylate motif. (C) 2006 American Institute of Physics. [DOI: 10.1063/1.2212423]

\section{INTRODUCTION}

Calcium ions control many biological functions by activating molecular switches in the cytoplasm. Therefore, cytosolic $\mathrm{Ca}^{2+}$ concentrations are actively maintained at submicromolar levels, and the molecular switches are activated when $\mathrm{Ca}^{2+}$ enters the cytoplasm from storage sites inside the cell or from the extracellular space. Both of these intracellu-

\footnotetext{
${ }^{a)}$ Author to whom correspondence should be addressed. Electronic mail: dezso_boda@rush.edu
}

lar and extracellular compartments contain 1-3 mM concentrations of free $\mathrm{Ca}^{2+}$ (i.e., $\mathrm{Ca}^{2+}$ not bound to proteins such as calsequestrin) and both are separated from the cytoplasm by a lipid membrane. $\mathrm{Ca}^{2+}$ crosses these particular membranes through molecular pores formed by channel proteins. When these ion channels open, they selectively conduct $\mathrm{Ca}^{2+}$ and therefore refered to as calcium $(\mathrm{Ca})$ channels. Selectivity for $\mathrm{Ca}^{2+}$ is vital for their biological function because most of the ions nearby are not $\mathrm{Ca}^{2+}$. The most abundant other ions are $\mathrm{Na}^{+}$(in the extracellular space) and $\mathrm{K}^{+}$(inside the cell and its compartments), typically both at concentrations of approxi- 
mately $150 \mathrm{mM}$. To conduct $\mathrm{Ca}^{2+}$, Ca channels must overcome a 100:1 unfavorable concentration ratio of monovalent ions to $\mathrm{Ca}^{2+}$.

Different Ca channels conduct different amounts of $\mathrm{Ca}^{2+}$ under identical conditions. For example, in single-channel recordings of current/voltage curves with $100 \mathrm{mM} \mathrm{Na}^{+}$or $\mathrm{K}^{+}$ on both sides of the membrane, a few micromolars of added $\mathrm{Ca}^{2+}$ is enough to reduce the monovalent current by half (compared to the current in $0 \mathrm{Ca}^{2+}$ ) in the $L$-type Ca channel. On the other hand, it takes 1000 times as much $\mathrm{Ca}^{2+}$ (a few millimolars) to have the same effect in the ryanodine receptor (RyR) Ca channel. ${ }^{1}$ Although it is not practical to experimentally measure the different ionic compositions of the $L$-type or RyR currents, the measureable change in the net current as bath $\mathrm{Ca}^{2+}$ concentration increases from zero indicates that $\mathrm{Ca}^{2+}$ current increases as well. This change in the ionic components of current must reflect changing channel occupancies of $\mathrm{Ca}^{2+}$ and, for example, $\mathrm{Na}^{+}$. Here we use equilibrium Monte Carlo (MC) simulations to study channel occupancy (as bath $\mathrm{Ca}^{2+}$ concentration increases) to assess the "affinity" of our model channel for $\mathrm{Ca}^{2+}$.

The different selectivities of the $L$-type Ca channel and RyR for $\mathrm{Ca}^{2+}$ are important for the different physiological functions of these channels. For example, the $L$-type $\mathrm{Ca}$ channel of cardiac and skeletal muscle controls $\mathrm{Ca}^{2+}$ movement from the extracellular compartment. The action of the $L$-type channel opens RyR that controls the release of $\mathrm{Ca}^{2+}$ from intracellular stores. This large release of $\mathrm{Ca}^{2+}$ over several milliseconds initiates muscle contraction. The enormous selectivity of $L$-type $\mathrm{Ca}$ channels allows $\mathrm{Ca}^{2+}$ to dominate in the pore and thereby preclude-in effect "block"- the flux of monovalent ions such as $\mathrm{Na}^{+}$through the $L$-type channel under physiological conditions. ${ }^{2}$ On the other hand, a model of ion permeation through RyR (Ref. 3) has shown that the weaker selectivity of RyR allows conduction of both $\mathrm{Ca}^{2+}$ and $\mathrm{K}^{+}$under the same conditions. Conduction of $\mathrm{Ca}^{2+}$ out of the intracellular stores with simultaneous conduction of $\mathrm{K}^{+}$ in the opposite direction may be one way for RyR to keep the electrostatic potential across the channel near zero and thus sustain $\mathrm{Ca}^{2+}$ release over several milliseconds. ${ }^{4}$

Mutation studies on both the $L$-type Ca channel and RyR have identified the amino acids primarily responsible for the $\mathrm{Ca}^{2+}$ selectivity of these channels. In the $L$-type $\mathrm{Ca}$ channel, four glutamates (the EEEE locus) were found essential for selectivity, ${ }^{5-7}$ while in RyR, four aspartates were identified that give RyR a DDDD locus. ${ }^{8}$ (In RyR, two other amino acids - one glutamate and one aspartate-also affect $\mathrm{Ca}^{2+}$ selectivity, but to a lesser extent. ${ }^{9,10}$ ) These glutamates and aspartates are similar amino acids with a carboxylate group $\left(\mathrm{COO}^{-}\right)$at the end of the side chain. Therefore, both the $L$-type Ca channel and RyR have carboxylate-rich selectivity filters, but very different $\mathrm{Ca}^{2+}$ versus monovalent selectivities. This paper addresses how similar selectivity filters can produce significantly different $\mathrm{Ca}^{2+}$ affinities by examining how the polarization properties of the protein affect $\mathrm{Ca}^{2+}$ affinity.

Carboxylate-rich loci have also been observed in proteins other than ion channels. Many $\mathrm{Ca}^{2+}$-selective proteins have $\mathrm{Ca}^{2+}$ binding sites in cavities lined by oxygen atoms ${ }^{11}$ from carboxylate groups of amino acids. In addition to carboxylate groups, in the EF-hand motif observed in many $\mathrm{Ca}^{2+}$ binding proteins, ${ }^{12}$ oxygens of hydroxyl or carbonyl groups and a water molecule are also involved in coordinating $\mathrm{Ca}^{2+}$. Like $\mathrm{Ca}$ channels, proteins with EF-hand motifs (for which crystallographic information exists ${ }^{13}$ ) have different $\mathrm{Ca}^{2+}$ affinities. ${ }^{12}$

The nature of the high $\mathrm{Ca}^{2+}$ affinity of the $L$-type $\mathrm{Ca}$ channel is a matter of debate. ${ }^{14-24}$ In order to quantify the various energy terms, Nonner and Eisenberg used an extended Poisson-Nernst-Planck (PNP) description of $L$-type $\mathrm{Ca}$ channels to estimate the excess chemical potentials needed to produce the experimentally observed specificities. ${ }^{22}$ Subsequent calculations using only bulk electrolyte theories ${ }^{18,21}$ showed that the excess chemical potentials needed to produce selectivity could arise from simple physical effects. Correlations produced by the finite diameter of the ions accounted for most of the excess free energy producing selectivity; no other chemical forces were necessary. We have also shown that such calculations with bulk solutions are qualitatively (and often quantitatively) accurate when compared to results computed in confining channel geometries. ${ }^{25}$ The reduced model of the Ca channel that was developed from this theoretical work was reexamined using MC simulations, similar to the simulations in this paper. $^{16,17,20}$

The results of all these works led to the hypothesis that Ca channel selectivity is produced, to first order, by a competition of charged ions for room in a crowded space [the charge/space competition (CSC) mechanism]. In recent years, CSC has been successfully tested in several experimental situations: (1) by engineering $\mathrm{Ca}^{2+}$-selective channels through modification of the OmpF porin; ${ }^{26,27}$ (2) by conferring $\mathrm{Ca}^{2+}$ sensitivity to pores in abiotic plastic membranes; ${ }^{28}$ and (3) by reproducing - and predicting - a wide range of experimental data from RyR with alkali metal and alkali earth cations ${ }^{3}$ using a CSC-based theory that combined PNP and density functional theory (DFT) of fluids. ${ }^{29,30}$ The success of this RyR modeling supports both the CSC mechanism and the idea that the movement of finite size ions through the pore can be described by the basic equations of electrodiffusion (PNP) without additional specific chemical forces.

Here we continue our study of model Ca channels with equilibrium calculations of channel occupancy. To make the complex link between occupancy and experimentally measureable currents requires a theory of ion permeation, for example, PNP/DFT (Refs. 29 and 30) or Brownian dynamics. ${ }^{14,15,19,23,24}$ However, studying only ion accumulation in a channel at equilibrium reduces the complexity of the problem and allows us to use the grand canonical (GC) ensemble to simulate micromolar $\mathrm{Ca}^{2+}$ concentrations.

In our study, we determine the binding affinity of our model $\mathrm{Ca}$ channel as the dielectric properties of the protein are changed. We show that changes in the dielectric environment can shift $\mathrm{Ca}^{2+}$ affinities from the millimolar range into the micromolar range. This suggests that adjusting the dielectric environment of the surrounding protein is a possible 


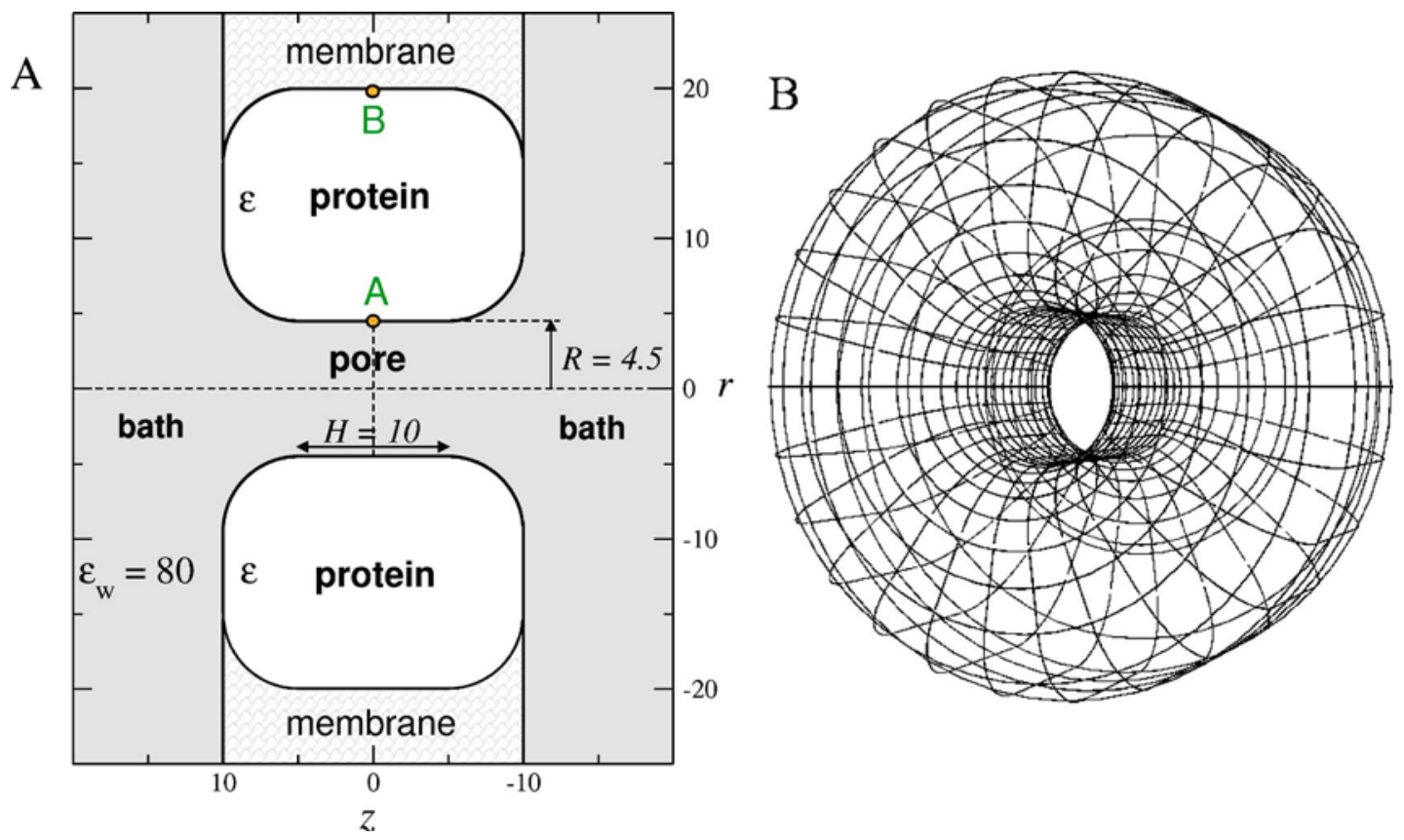

FIG. 1. (Color online) (A) Cross section of the cylindrical simulation cell. Two "bath" regions are connected via a molecular "pore," a central cylinder flanked by vestibules. The "protein" forming the pore is a doughnut-shaped body with rounded edges. The protein is embedded in a "membrane" that separates the two baths. The figure is drawn to scale (units in $\AA$ ). (B) Three-dimensional view of the protein surface. The surface grid outlines the "tiles" of the discretized surface used in the electrostatics computations.

tool that allows evolution to regulate the $\mathrm{Ca}^{2+}$ specificity of the common carboxylate motif, although we do not believe it to be the only such tool.

Dielectric properties of channel walls have previously been considered, ${ }^{31-35}$ most recently by Chung et al. in Brownian dynamics studies of ion conduction through $\mathrm{Ca}$ channels. ${ }^{14,15,19,23,24}$ The lowest bath concentration of $\mathrm{Ca}^{2+}$ tested in their simulations was $18 \mathrm{mM}$ (corresponding to one $\mathrm{Ca}^{2+}$ in the system). Affinity in the micromolar range of bath $\mathrm{Ca}^{2+}$ concentration was estimated by an extrapolation over a range of $10^{4}$, from 18 to $0.001 \mathrm{mM}$ based on kinetics. Here, affinity in the micromolar range was simulated directly with bath $\mathrm{Ca}^{2+}$ concentrations from 10 to $0.012 \mathrm{mM}$-without extrapolation-using the GC ensemble.

The dielectric inhomogeneity that appears at the boundary of the electrolyte solution and the protein raises substantial numerical difficulties regarding both the accuracy and efficiency of the method used to calculate the electrostatics. With regard to accuracy, the dependence of results on the resolution of the numerical calculations and problems due to curved dielectric boundaries had to be checked, while with regard to efficiency, the electrostatic energy had to be updated in every simulation step. We deal with these difficulties by using the induced charge computation (ICC) method. ${ }^{36}$ We discuss the method and show its accuracy in the channel geometry considered in this work.

\section{MODEL}

We simulate a $\mathrm{Ca}$ channel containing the fourcarboxylate motif and ions of an electrolyte bath in a reduced model. Figure 1(a) shows a central cross section of the simulation cell; the three-dimensional (3D) geometry is obtained by rotating one-half of Fig. 1(a) around the axis defined by $r=0$ [Fig. 1(b)]. There is also symmetry with respect to the plane $z=0$ that allows us to average results obtained in corresponding positions of both half-cells. The protein forming the wall of the aqueous pore and the cell membrane surrounding the protein are represented as a region that is bounded by hard walls. The protein surface forms a sharp dielectric boundary that encloses a region of (varied) dielectric coefficient $\epsilon$ and separates it from the uniform dielectric in the rest of the cell (assigned the dielectric coefficient 80, a value characteristic of aqueous solutions). We use a dielectric coefficient of 80 (rather than 2) for the membrane region to reduce the computational effort for the electrostatics; controls showed no significant effect on the results of interest (see Fig. 1 of the supplementary material ${ }^{37}$ ).

The four carboxylate groups of the protein channel are modeled as eight half-charged anions with a radius of $1.4 \AA$ that are restricted to the cylindrical part of the pore (radius $R=4.5 \AA$ and length $H=10 \AA$ ) but are free to move within this region. These "oxygen ions" are confined within the cylindrical pore region $(|z| \leqslant 3.6 \AA)$ where they are not allowed to overlap with the wall of the pore $(r \leqslant 3.1 \AA)$.

Ions $\left(\mathrm{Na}^{+}, \mathrm{Ca}^{2+}\right.$, and $\left.\mathrm{Cl}^{-}\right)$are modeled as charged hard spheres $\left(r_{\mathrm{Na}}=1 \AA, r_{\mathrm{Ca}}=0.99 \AA\right.$, and $\left.r_{\mathrm{Cl}}=1.81 \AA\right)$ and are allowed to distribute within the "solution" region, which includes the pore and the two baths. The solvent is represented as a uniform dielectric with a dielectric coefficient of 80 . Ions are confined to the simulation cell by hard walls, but $\mathrm{CaCl}_{2}$ salt can be exchanged against a large reservoir of a chosen chemical potential, as detailed below. The dimensions of the bath regions are large enough to allow the formation of bulklike electrolytes in the centers of the baths. 
The aqueous dielectric extends into the space outside the cell. The simulation includes a single, rather than periodically replicated, simulation cell.

\section{METHODS}

\section{A. Monte Carlo simulations}

We use the standard Metropolis algorithm ${ }^{38-40}$ in a mixed ensemble. The volume of the simulation cell $V$ and the temperature $T=298 \mathrm{~K}$ are constant, the number of $\mathrm{Na}^{+}$is fixed, and the numbers of $\mathrm{Ca}^{2+}$ and $\mathrm{Cl}^{-}$are allowed to fluctuate as in a grand canonical ensemble. Three kinds of MC steps are used.

(1) Simple particle displacement. We attempt to displace a randomly chosen ion to a randomly chosen position in two ways: (a) within a fixed neighborhood of its original position (useful in sampling high-density regions like the pore) or (b) anywhere in the simulation cell (useful in sampling lowdensity regions like the bath). In both cases, the acceptance probability is $p=\min [1, \exp (-\Delta U / k T)]$, where $k$ is the Boltzmann constant and $\Delta U$ is the energy change of the system in the MC step.

(2) Biased particle displacement between pore and bath. When only simple displacements are allowed, ions rarely move between the pore and the baths because (1) a particle rarely attempts to leave the pore because the number of particles in the pore is small, and (2) a particle rarely attempts to enter the pore because the volume of the pore is small. To improve sampling and accelerate convergence of the average number of different ions in the pore, we move a randomly chosen ion located in the pore to the bath, or vice versa. The bias inherent to this kind of displacement is accounted for by the acceptance probability

$$
p^{\text {bias }}=\min \left[1, \frac{V_{\text {new }}}{V_{\text {old }}} \exp \left(-\frac{\Delta U}{k T}\right)\right],
$$

where $V_{\text {new }}$ and $V_{\text {old }}$ are the volumes of the two compartments between which the ion is moved. ${ }^{16}$

(3) Particle insertion/deletion. Because we wish to study $\mathrm{Ca}^{2+}$ absorption from a very dilute $\mathrm{Ca}^{2+}$ bath, we equilibrate $\mathrm{CaCl}_{2}$ between the simulation cell and a reservoir of given chemical potential. Following the grand canonical method of Ref. 41, we insert $1 \mathrm{Ca}^{2+}$ and $2 \mathrm{Cl}^{-}$at random positions in the simulation cell or we delete $1 \mathrm{Ca}^{2+}$ and $2 \mathrm{Cl}^{-}$that are randomly chosen from the simulation cell, subject to the acceptance probability

$p^{\mathrm{GC}}=\min \left[1,\left(\frac{N_{\mathrm{Ca}} ! N_{\mathrm{Cl}} !}{\left(N_{\mathrm{Ca}}+\nu\right) !\left(N_{\mathrm{Cl}}+2 \nu\right) !}\right)^{\nu} \exp \left(\frac{\nu B-\Delta U}{k T}\right)\right]$,

with

$$
B=\mu_{\mathrm{Ca}}+2 \mu_{\mathrm{Cl}}+\ln \frac{V_{\mathrm{Ca}}}{\lambda_{\mathrm{Ca}}^{3}}+2 \ln \frac{V_{\mathrm{Cl}}}{\lambda_{\mathrm{Cl}}^{3}},
$$

and

$$
\lambda_{i}=\frac{h}{\left(2 \pi m_{i} k T\right)^{1 / 2}} .
$$

$N_{\mathrm{Ca}}$ and $N_{\mathrm{Cl}}$ are the numbers of the ions in the cell before the operation, $\mu_{i}$ is the chemical potential, and $m_{i}$ is the mass of the ionic species $i . V_{\mathrm{Ca}}$ and $V_{\mathrm{Cl}}$ are the volumes of the cell region accessible to the respective ionic species. The value of $\nu$ is 1 for insertion and -1 for deletion. Choosing a value for $B$ uniquely defines the grand canonical step and the average bath $\mathrm{Ca}^{2+}$ concentration.

Both insertion and deletion of $\mathrm{CaCl}_{2}$ are attempted in $25 \%$ of the trials. In the other half of the trials, an ionic species is chosen at random and simple or biased displacements are attempted with equal weights. Trials are partitioned this way to speed up convergence; the partitioning does not affect the final result of the MC simulation. ${ }^{38-40}$ Only simple displacements are attempted for the structural oxygen ions that are confined within the pore cylinder.

The purpose of the simulations was to determine the pore's absorption of ions from a bath with $0.1 \mathrm{M} \mathrm{NaCl}$ and a variable amount of added $\mathrm{CaCl}_{2}$ for different dielectric coefficients $\epsilon$ of the protein $(2,5,10,20,40$, and 80). Appropriate cell dimensions were determined in preliminary simulations. A cell containing $300 \mathrm{Na}^{+}$in a cylinder $75 \AA$ in radius and $320 \AA$ in length gave nearly identical results as cells containing 200 or $400 \mathrm{Na}^{+}$in appropriate volumes (Fig. 3 of the supplementary material ${ }^{37}$ ); therefore, the cell with 300 $\mathrm{Na}^{+}$was used in production runs. The concentration of $\mathrm{Ca}^{2+}$ in the baths was varied between $12 \mu \mathrm{M}$ and $10 \mathrm{mM}$ by varying the value of the parameter $B$ in Eq. (2). In the absence of $\mathrm{Ca}^{2+}$, canonical simulations were done to determine the absorption of $\mathrm{Na}^{+}$from the pure $0.1 M \mathrm{NaCl}$ bath. The statistical errors indicated in graphs are the standard deviations of 20-99 runs starting from different seeds. Each run included $6 \times 10^{8}-9 \times 10^{8} \mathrm{MC}$ trials.

\section{B. Electrostatics}

The simulations involve the electrostatic energy of a system whose source charge with density $\rho(\mathbf{r})$ is embedded in nonuniform polarizable matter. The electrical field $\mathbf{E}$ including the polarization $\mathbf{P}$ is described by Poisson's equation,

$\epsilon_{0} \nabla \cdot \mathbf{E}(\mathbf{r})=\rho(\mathbf{r})-\nabla \cdot \mathbf{P}(\mathbf{r})$.

In our reduced model of the Ca channel, we describe the polarization by the linear constitutive relation

$$
\mathbf{P}(\mathbf{r})=(\epsilon(\mathbf{r})-1) \epsilon_{0} \mathbf{E}(\mathbf{r}),
$$

where $\epsilon(\mathbf{r})$ is the location-dependent dielectric coefficient. Using Eqs. (5) and (6), the polarization charge density $h(\mathbf{r})$ $=-\nabla \cdot \mathbf{P}(\mathbf{r})$ can be expressed as

$$
h(\mathbf{r})=\frac{1-\epsilon(\mathbf{r})}{\epsilon(\mathbf{r})} \rho(\mathbf{r})-\epsilon_{0} \frac{\nabla \epsilon(\mathbf{r})}{\epsilon(\mathbf{r})} \cdot \mathbf{E}(\mathbf{r}) .
$$

The first term of the right-hand side represents the charge induced on the surface of an infinitesimal volume element by the source charge contained in this volume element, and the second term represents the charge induced in the volume 
element by the electric field where the dielectric is spatially nonuniform.

In our simulations, the source charge is a set of point charges (located at the center of hard-sphere ions) so $\rho(\mathbf{r})$ $=\Sigma_{k} q_{k} \delta\left(\mathbf{r}-\mathbf{r}_{k}\right)$, where $q_{k}$ is the charge of the $k$ th point charge located at $\mathbf{r}_{k}$. Furthermore, the dielectrics in our simulations form regions of constant dielectric coefficient separated by a sharp boundary surface $\mathcal{B}$. Hence, charge is only induced on the infinitesimal surface of each (point) source charge $\left(h\left(\mathbf{r}_{k}\right)\right)$ and on the boundary surface $\mathcal{B}(h(\mathbf{s}))$,

$$
\begin{aligned}
h(\mathbf{r}) & =\delta\left(\mathbf{r}-\mathbf{r}_{k}\right) h\left(\mathbf{r}_{k}\right)+\delta(\mathbf{r}-\mathbf{s}) h(\mathbf{s}) \\
& =\delta\left(\mathbf{r}-\mathbf{r}_{k}\right) \sum_{k} \frac{1-\epsilon\left(\mathbf{r}_{k}\right)}{\epsilon\left(\mathbf{r}_{k}\right)} q_{k}-\delta(\mathbf{r}-\mathbf{s}) \epsilon_{0} \frac{\Delta \epsilon(\mathbf{s})}{\bar{\epsilon}(\mathbf{s})} \mathbf{n}(\mathbf{s}) \cdot \mathbf{E}(\mathbf{s}),
\end{aligned}
$$

where $\mathbf{s}$ is any location on $\mathcal{B}, \Delta \boldsymbol{\epsilon}(\mathbf{s})$ is the change of dielectric coefficient in the normal direction $\mathbf{n}(\mathbf{s})$ of the dielectric boundary, and $\bar{\epsilon}(\mathbf{s})$ is the mean of the dielectric coefficients at the boundary location. By Coulomb's law and superposition, the electric field at the boundary is

$$
\begin{aligned}
\mathbf{E}(\mathbf{s})= & \frac{1}{4 \pi \epsilon_{0}} \int_{\mathcal{B}} \frac{\mathbf{s}-\mathbf{s}^{\prime}}{\left|\mathbf{s}-\mathbf{s}^{\prime}\right|^{3}} h\left(\mathbf{s}^{\prime}\right) d \mathbf{s}^{\prime} \\
& +\frac{1}{4 \pi \epsilon_{0}} \sum_{k} \frac{q_{k}}{\epsilon\left(\mathbf{r}_{k}\right)} \frac{\mathbf{s}-\mathbf{r}_{k}}{\left|\mathbf{s}-\mathbf{r}_{k}\right|^{3}} .
\end{aligned}
$$

Inserting Eq. (9) into Eq. (8) yields the integral equation for $h(\mathbf{s})$,

$$
\begin{aligned}
h(\mathbf{s}) & +\frac{\Delta \boldsymbol{\epsilon}(\mathbf{s})}{4 \pi \bar{\epsilon}(\mathbf{s})} \mathbf{n}(\mathbf{s}) \cdot \int_{\mathcal{B}} \frac{\mathbf{s}-\mathbf{s}^{\prime}}{\left|\mathbf{s}-\mathbf{s}^{\prime}\right|^{3}} h\left(\mathbf{s}^{\prime}\right) d \mathbf{s}^{\prime} \\
& =-\frac{\Delta \boldsymbol{\epsilon}(\mathbf{s})}{4 \pi \bar{\epsilon}(\mathbf{s})} \mathbf{n}(\mathbf{s}) \cdot \sum_{k} \frac{q_{k}}{\epsilon\left(\mathbf{r}_{k}\right)} \frac{\mathbf{s}-\mathbf{r}_{k}}{\left|\mathbf{s}-\mathbf{r}_{k}\right|^{3}} .
\end{aligned}
$$

When the integral equation in $h(\mathbf{s})$ is solved on the twodimensional boundary $\mathcal{B}$, all source and induced charges of the system are known and the electrical field at any location can be computed using Coulomb's law and superposition. This method of determining the electrical field is computationally more efficient than solving Poisson's equation in three dimensions.

This ICC method has been derived previously ${ }^{36,42}$ from the variational approach of Allen et al. ${ }^{43-46}$ Integral equation methods have been used, e.g., in work computing apparent surface charges (ASC) in polarizable continuum models (PCM) of solvation of macromolecules. ${ }^{47-52}$ Reducing the surface integral equation to a system of linear equations yields the boundary element method ${ }^{53-57}$ (BEM) also used by Green and $\mathrm{Lu}^{58,59}$ and $\mathrm{Lu}$ and $\mathrm{Green}^{60}$ in their simulations of ion channels.

In our simulations, ions (source charges) move within a uniform region of dielectric (the pore lumen and bath) but do not move between regions with different dielectrics. The part of the electrostatic energy that varies due to such a relocation of a source charge is

$$
U_{\mathrm{ES}}=\frac{1}{2} \sum_{k} \frac{q_{k}}{4 \pi \epsilon_{0}}\left(\sum_{j \neq k} \frac{q_{j}}{\epsilon\left(\mathbf{r}_{j}\right)\left|\mathbf{r}_{k}-\mathbf{r}_{j}\right|}+\int_{\mathcal{B}} \frac{h(\mathbf{s})}{\left|\mathbf{s}-\mathbf{r}_{k}\right|} d \mathbf{s}\right) .
$$

\section{Discretization of the dielectric boundary}

The simulation of the ion channel system involves dielectric boundaries that are curved (Fig. 1). In evaluating the surface integral of Eq. (10), the surface curvature and the spatial variation of the surface charge create numerical difficulties, and the resulting algorithms and codes have to be checked carefully for accuracy and efficiency in our experience. We discretize the dielectric boundary surface as a set of two-dimensional boundary elements called "tiles" $\mathcal{B}_{\alpha}$ of generally nonuniform size. The charge induced on each tile $h_{\alpha}$ is approximated as uniform over the tile and is computed from the field strength acting in the normal direction $\mathbf{n}_{\alpha}=\mathbf{n}\left(\mathbf{s}_{\alpha}\right)$ at the center of charge $\mathbf{s}_{\alpha}$ of the tile's area $a_{\alpha}$.

Discretizing Eq. (10) this way yields the system of linear equations

$$
\sum_{\beta} h_{\beta}\left[\delta_{\alpha \beta}+\frac{\Delta \epsilon_{\alpha}}{4 \pi \bar{\epsilon}_{\alpha}} I_{\alpha \beta}\right]=-\frac{\Delta \epsilon_{\alpha}}{4 \pi \bar{\epsilon}_{\alpha}} \sum_{k} \frac{q_{k}}{\epsilon\left(\mathbf{r}_{k}\right)} \frac{\left(\mathbf{s}_{\alpha}-\mathbf{r}_{k}\right)}{\left|\mathbf{s}_{\alpha}-\mathbf{r}_{k}\right|^{3}} \cdot \mathbf{n}_{\alpha},
$$

with

$$
I_{\alpha \beta}=\int_{\mathcal{B}_{\beta}} \frac{\left(\mathbf{s}_{\alpha}-\mathbf{s}^{\prime}\right) \cdot \mathbf{n}_{\alpha}}{\left|\mathbf{s}_{\alpha}-\mathbf{s}^{\prime}\right|^{3}} d \mathbf{s}^{\prime},
$$

where $\Delta \epsilon_{\alpha}=\Delta \epsilon\left(\mathbf{s}_{\alpha}\right), \bar{\epsilon}_{\alpha}=\bar{\epsilon}\left(\mathbf{s}_{\alpha}\right)$, and $\delta_{\alpha \beta}$ is the Kronecker delta function. These equations can be written in matrix notation as

$$
\mathrm{A} \mathbf{h}=\mathbf{c},
$$

where each element of the matrix $A$ is given by the expression in square brackets in Eq. (12), each element of the column vector $\mathbf{h}$ is given by $h_{\beta}$, and each element of the column vector $\mathbf{c}$ is given by the right-hand side of Eq. (12). For a given configuration of source charges, the right-hand side of Eq. (12) is known. Hence, the system can be solved for the induced charge densities $\mathbf{h}$. Since the matrix $A$ is dense, we use LU decomposition. This decomposition becomes part of the simulation overhead because A does not change when source charges are moved in the cell. The system can be solved for varied positions (and numbers) of the source charges by backsubstitution.

The discretized formula for the electrostatic energy is

$$
U_{\mathrm{ES}}=\frac{1}{2} \sum_{k} \frac{q_{k}}{4 \pi \epsilon_{0}}\left(\sum_{j \neq k} \frac{q_{j}}{\epsilon\left(\mathbf{r}_{j}\right)\left|\mathbf{r}_{k}-\mathbf{r}_{j}\right|}+\sum_{\beta} h_{\beta} J_{\beta}\right),
$$

with

$$
J_{\beta}=\int_{\mathcal{B}_{\beta}} \frac{d \mathbf{s}}{\left|\mathbf{s}-\mathbf{r}_{k}\right|}
$$




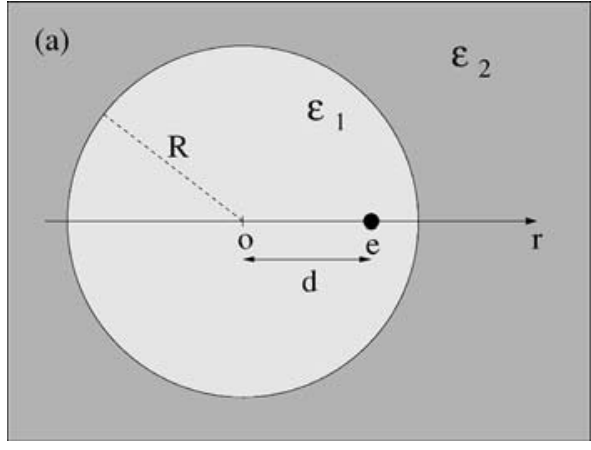

FIG. 2. Geometry used for assessing boundary discretizations. A spherical dielectric domain $\left(R=5 \AA, \epsilon_{1}=80\right)$ is embedded in a space containing a different dielectric $\left(\epsilon_{2}=2\right)$. An elementary point charge is placed inside the sphere at a distance $d=4 \AA$ from the center.

\section{Choosing discretization parameters}

The accuracy of the boundary discretization depends on both the choice of tile size and on the method of evaluation of the integrals $I_{\alpha \beta}$ and $J_{\beta}$. The tile size needs to be chosen small enough so that the electric field (and hence induced charge density) are sufficiently uniform within the area of the tile. The integration method is particularly important when the surface is curved. Then, charge induced on the same tile contributes to the normal electric field in the center of the tile, and these contributions are large because of the short distances. Nearby charge can induce charge on a tile as well. We have determined the tile size and evaluated different approximations to the integrals using the example of a point charge eccentrically located inside a dielectric sphere (Fig. 2). This example involves surface curvature and inhomogeneity of induced charge. Both are important. An analytical solution in terms of Legendre polynomials is known. ${ }^{61}$ We consider a sphere radius $(5 \AA)$ and dielectric coefficients $(80$ and 2) that are relevant for our simulations. Figure 3 plots

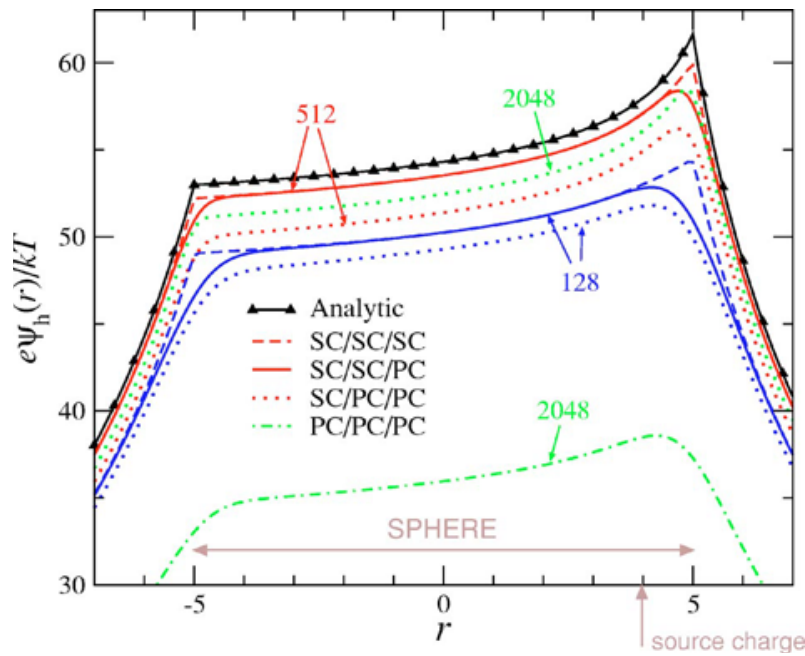

FIG. 3. (Color online) Accuracy of different boundary discretizations in the electrostatic system of Fig. 2. The potential produced by the charge induced on the sphere is plotted along the line connecting sphere center and point charge. The dielectric boundary is discretized into a varied number of surface tiles (the respective curves are labeled by the number of tiles) and different methods of evaluating the integrals $I_{\alpha \beta}$ and $J_{\beta}$ (curves distinguished by line patterns, for an explanation of the notation see text). The analytical result is shown as the black, solid line. analytical and numerically computed potentials for a case in which the source charge is located $1 \AA$ from the boundary. The potential is that generated by the charge induced on the sphere, along the straight line connecting source charge and sphere center (Fig. 2). The interaction of the source charge with its own induced charge is given by the potential at the position $r=4 \AA$.

In a first approximation to the integrals $I_{\alpha \beta}$ and $J_{\beta}$, the surface charge of the tile is represented as a point charge of magnitude $h_{\alpha} a_{\alpha}$ located at the center of the tile area. Furthermore, tiles are assumed to be flat so that $I_{\alpha \alpha}=0$. We call this the point charge (PC) approximation. In a second approximation, the tile is divided into $n \times n$ subtiles [ $n=10$ was used to compute $I_{\alpha \beta}(\alpha \neq \beta)$ and $n=20$ was used to compute $\left.I_{\alpha \alpha}\right]$. These follow the curvature of the tile and are assigned the same uniform induced charge density of the larger tile. The method of subtiling is detailed for the generalized cylinder geometry in the supplementary material. ${ }^{37}$ In this method, $I_{\alpha \alpha} \neq 0$ contributes a substantial term due to surface curvature. We call the second method the surface charge (SC) approximation.

The potential curves plotted in Fig. 3 were obtained using these methods in different combinations. The label SC/ $\mathrm{PC} / \mathrm{PC}$ indicates that the SC method was used in evaluating $I_{\alpha \alpha}$, and the PC method in both $I_{\alpha \beta}(\beta \neq \alpha)$ and $J_{\beta}$. The potential computed with $\mathrm{PC} / \mathrm{PC} / \mathrm{PC}$ strongly deviates from the analytical result even though the spherical boundary was discretized into a particularly large number of tiles (2048). The SC/SC/SC method yields far more accurate results with a discretization of 512 tiles; it is more accurate than PC/ $\mathrm{PC} / \mathrm{PC}$ even when the number of tiles is reduced from 2048 tiles to 128 . SC/SC/SC with 2048 tiles is indistinguishable from the analytic curve. SC/SC/PC yields accurate potentials up to positions less than $1 \AA$ away from the dielectric boundary. Since ions are excluded from this region in our simulations, we use the $\mathrm{SC} / \mathrm{SC} / \mathrm{PC}$ method in production runs. Using the PC method in the computations of energy avoids additional computational cost in evaluating $J_{\beta}$ whenever an ion is moved in the simulation.

Finally, we note that $\mathrm{SC} / \mathrm{PC} / \mathrm{PC}$ produces a larger error than $\mathrm{SC} / \mathrm{SC} / \mathrm{PC}$. Hence, using the SC method is essential in evaluating $I_{\alpha \alpha}$, but using the SC method in evaluating $I_{\alpha \beta}$ $(\beta \neq \alpha)$ still gives a substantial reduction of error compared to the PC method. Correction for boundary curvature have been devised in many implementations of the BEM to consider the effects of charge induced on the same surface element $I_{\alpha \alpha}$ (Refs. 23, 47, 49, 53, and 62) (see also the reviews ${ }^{63,64}$ ). Our approximation is based on direct numerical integration rather than analytical corrections based on approximations. We find it necessary to apply the numerical integration also to compute charges induced by the field of other surface elements.

\section{Tests of numerical accuracy}

No analytical solution is known for the electrostatics of the system that we simulate (Fig. 1). Nevertheless, an independent test of accuracy is possible using Gauss's law. The dielectric domain of the protein in our simulations contains no source charges. By Gauss's law, the total charge induced 


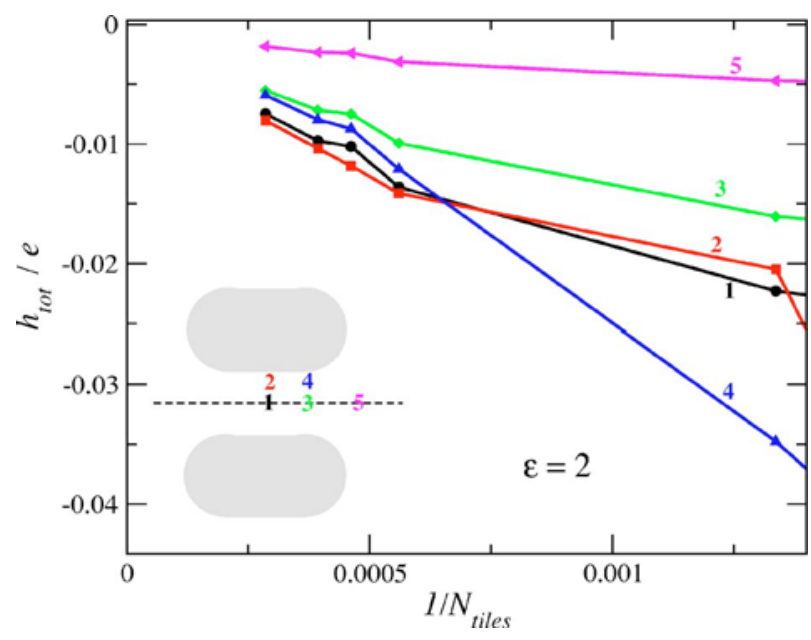

FIG. 4. (Color online) Sum rule test based on Gauss's law. An elementary point charge is placed at various locations (see inset) in or near the pore of the simulation cell [compare Fig. 1(a)]. Points 1, 3, and 5 are on the centerline, while points 2 and 4 are $1 \AA$ from the pore wall. Points 1 and 2 are in the middle in the pore, while points 3 and 4 are at the entrance of the pore. Point 5 is in the solution near the pore. The charge induced on the protein surface has been integrated and plotted vs the inverse number of tiles used in the surface discretization. The exact result from Gauss's law is that the charge induced on the protein is zero because the protein region does not enclose any source charges (Ref. 61).

by ions on the closed surface of the protein must equal zero. Figure 4 plots computed total surface charges of the protein as a function of the inverse number of tiles used in the discretization of the boundary. The charge on the boundary was induced by a single source charge (1e) placed at five different test locations, as shown by the inset of Fig. 4.

In a second kind of test, MC runs with the actual simulation cell (Fig. 1) were done using discretizations of 540, 690, and 1462 tiles (Fig. 2 of supplementary material ${ }^{37}$ ). Concentration profiles were virtually indistinguishable for all choices. Production runs were with the discretization of 690 tiles. The dimension of these tiles was chosen smallest for the wall of the pore $(\approx 1 \AA)$ and gradually increased to $\approx 5 \AA$ in the periphery of the protein [see Fig. 1(b)]. This choice of discretization explains why the errors in the total induced charge seen in Fig. 4 have little effect on the simulation results (see Fig. 2 of supplementary material ${ }^{37}$ ). Errors are mainly due to the coarser discretization of boundary regions far from the pore, which has small effects on the ions in the space of interest, that is, in and near the pore.

\section{RESULTS AND DISCUSSION}

The main results of the simulations are the ionic concentration profiles. We first consider whether variation of the protein's dielectric has qualitative effects on the packing of ions and structural charges in the pore. The longitudinal, cross sectionally averaged profiles of $\mathrm{Ca}^{2+}, \mathrm{Na}^{+}$, and oxygen ion concentrations for different dielectric coefficients are plotted in Fig. 5. The structural charges do not rearrange over the range $2 \leqslant \epsilon \leqslant 80$ and the mobile ions also distribute in a stable pattern as $\epsilon$ is varied. The dielectric coefficient, however, controls the absolute concentrations of the mobile ions. Although both $\mathrm{Ca}^{2+}$ and $\mathrm{Na}^{+}$are strongly absorbed into the pore, $\mathrm{Ca}^{2+}$ is absorbed over $\mathrm{Na}^{+}$as the dielectric coefficient

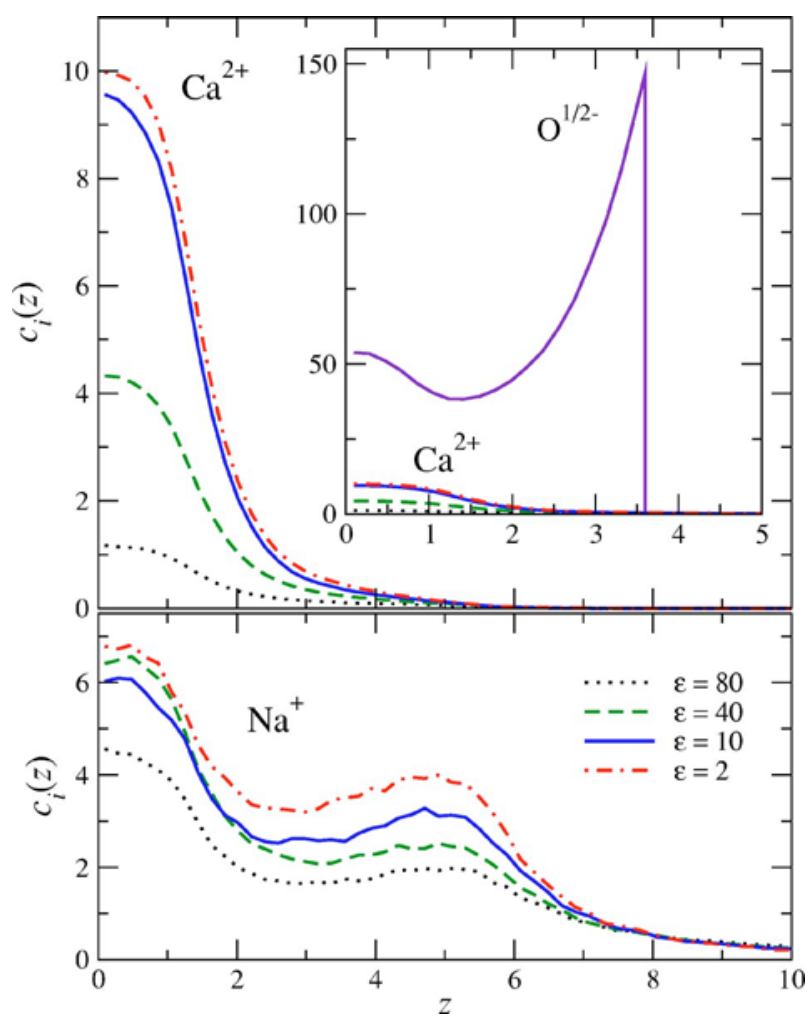

FIG. 5. (Color online) Longitudinal packing of mobile and structural ions in the pore. The concentration profiles are averaged over the cross section. The bath contains $8.6 \times 10^{-5} M \mathrm{CaCl}_{2}$ and $0.1 M \mathrm{NaCl}$.

is reduced. Unlike $\mathrm{Ca}^{2+}, \mathrm{Na}^{+}$absorption increases more strongly near the pore mouths than at the pore center.

The radial distributions of the mobile and structural ions also only undergo gradual quantitative variations as the dielectric coefficient is varied. Figure 6 shows concentration profiles in both the axial and radial dimensions of the pore for $\epsilon=10$. The oxygen ions dwell near the pore walls, except at the ends of the pore, where they spread more evenly over the cross section. The central space of the pore is occupied by $\mathrm{Ca}^{2+}$ and $\mathrm{Na}^{+}$that are screened in this way by a shell of oxygen ions. $\mathrm{Ca}^{2+}$ always packs near the pore axis, whereas $\mathrm{Na}^{+}$is more spread out over the cross section. The same pattern is seen when $\epsilon=80$ (not shown). Overall, the packing of the particles in the pore is not qualitatively changed by varying the protein dielectric. It appears that the presence of the dielectric boundary more or less enhances a pattern of packing that is already stable due to screening and excludedvolume interactions among the charged particles in the pore.

To present our simulation results for different $\epsilon$ in a concise way, we distill the concentration profiles into a single number, the occupancy of an ionic species. For each ionic species, the affinity of the channel for that species is characterized by its occupancy, while the selectivity is characterized by the relative occupancies of the two ionic species. For this we have to define the region of the pore $|z| \leqslant \Delta H / 2$ over which we integrate the ionic profiles to calculate the average number of each ionic species. Figure 5 shows that $\mathrm{Ca}^{2+} \mathrm{ac}-$ cumulates in the center of the pore, while $\mathrm{Na}^{+}$accumulates both in the center of the pore and in the atria. Therefore the choice of $\Delta H$ will affect the calculated occupancy. One 

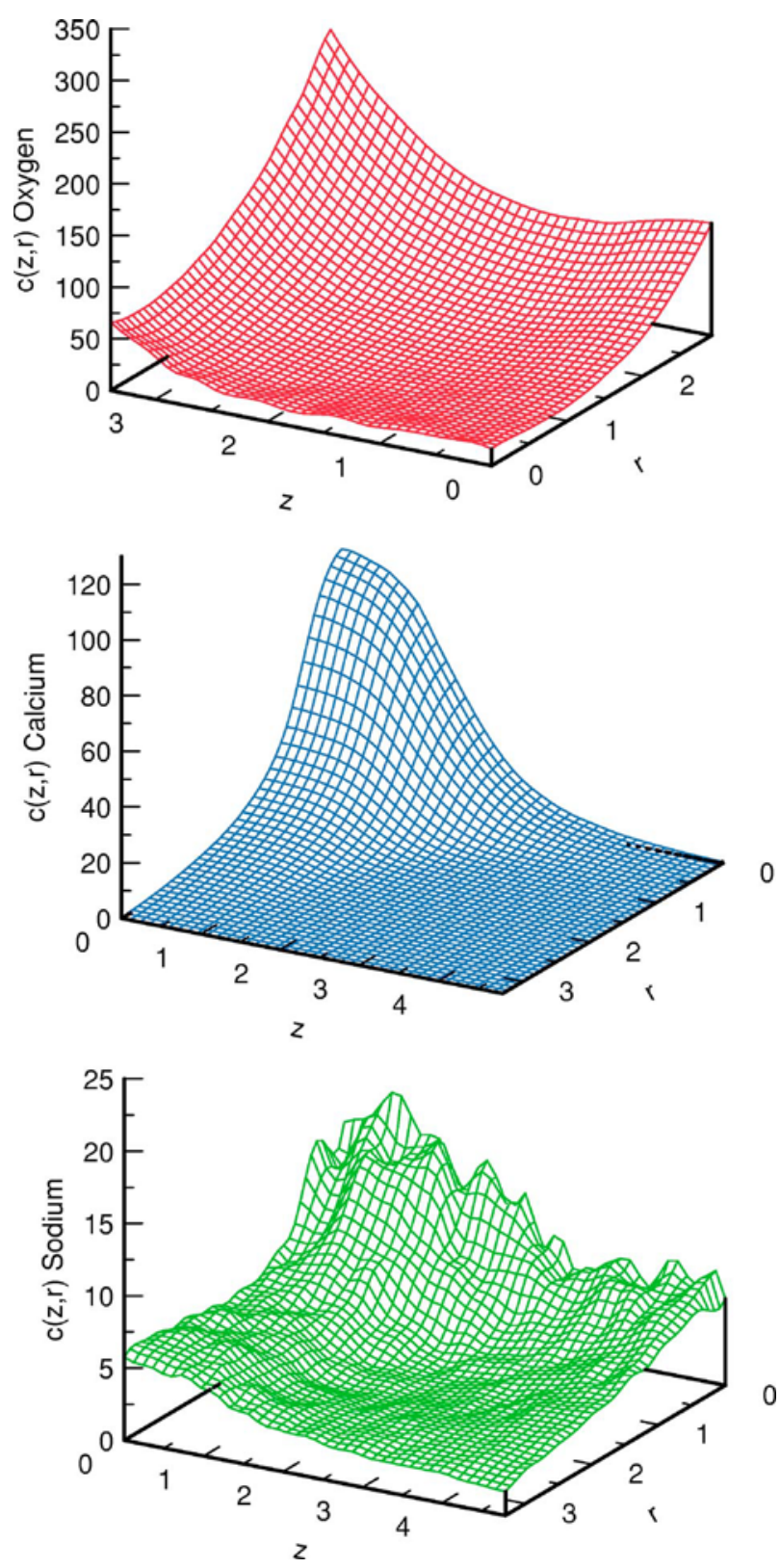

FIG. 6. (Color online) Axial and radial average distributions of ions in the pore. The bath contains $8.6 \times 10^{-5} M \mathrm{CaCl}_{2}$ and $0.1 M \mathrm{NaCl}$; the protein's dielectric coefficient is 10 .

choice is the whole length of the cylindrical pore $(\Delta H$ $=10 \AA)$. We also consider the central region of the pore $(\Delta H=4.2 \AA)$, where $\mathrm{Ca}^{2+}$ accumulates more favorably than $\mathrm{Na}^{+}$as $\epsilon$ is decreased. Figure 7 shows the average number of $\mathrm{Ca}^{2+}$ and $\mathrm{Na}^{+}$accumulated in the whole pore cylinder $(10 \AA$ long) and in the central pore region ( $4.2 \AA$ long) for two fixed $\mathrm{CaCl}_{2}$ concentrations as functions of the dielectric coefficient of the protein. The accumulations in the two regions are similar for $\mathrm{Ca}^{2+}$, which is concentrated in the central region. The accumulations of $\mathrm{Na}^{+}$differ more substantially between the two regions because $\mathrm{Na}^{+}$also accumulates in the pore region outside the central region.

The competitive accumulation of counterions in the charged pore defines the selectivity of the system, a measure of immediate biological interest. Figure 8 shows, for each protein dielectric coefficient that was tested, the number of

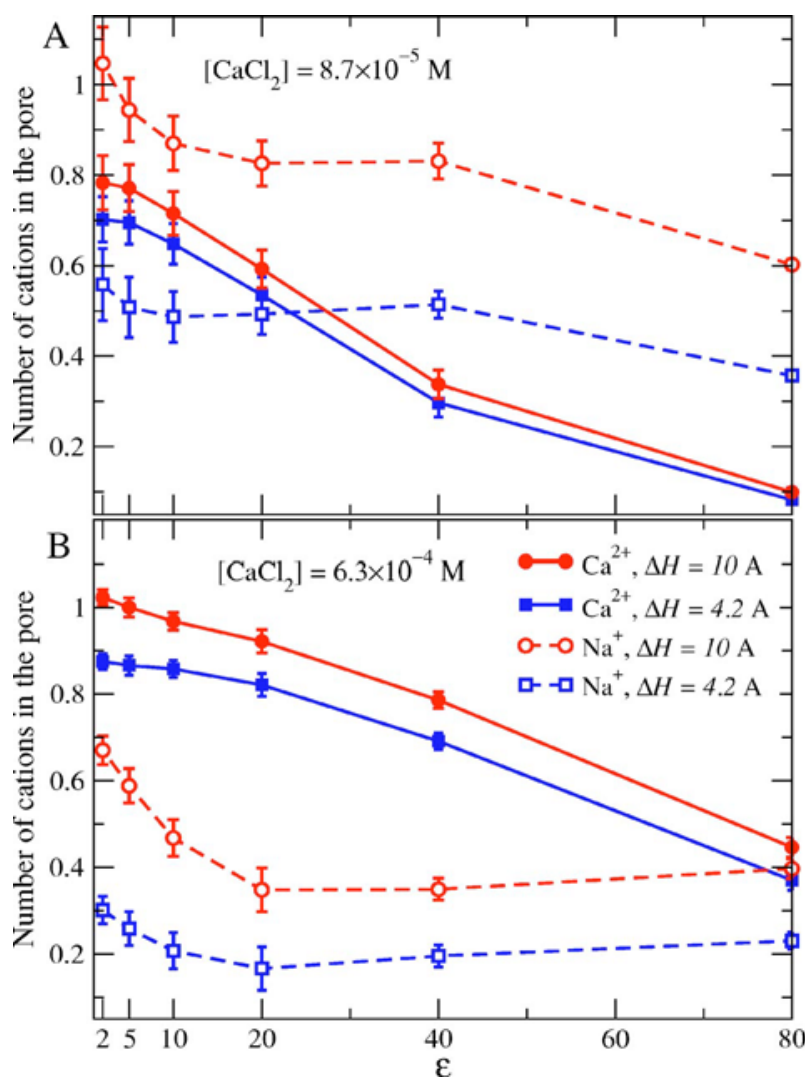

FIG. 7. (Color online) Effect of protein dielectric coefficient on the absorption of ions in the pore. Numbers of $\mathrm{Ca}^{2+}$ and $\mathrm{Na}^{+}$accumulated in the pore cylinder $(\Delta H=10 \AA)$ or in the central region of the pore cylinder $(\Delta H$ $=4.2 \AA$ ) are given for two bath compositions including the given concentrations of $\mathrm{CaCl}_{2}$ and $0.1 M \mathrm{NaCl}$.

$\mathrm{Ca}^{2+}$ and $\mathrm{Na}^{+}$accumulated in the pore cylinder $(\Delta H$ $=10 \AA$ ) from baths containing $0.1 \mathrm{M} \mathrm{NaCl}$ and a varied concentration of $\mathrm{CaCl}_{2}$ (curves calculated with $\Delta H=4.2 \AA$ are seen in Fig. 4 of the supplementary material ${ }^{37}$ ). Reducing the dielectric coefficient of the protein enhances the accumulations of both ions, $\mathrm{Ca}^{2+}$ and $\mathrm{Na}^{+}$. The bath $\mathrm{CaCl}_{2}$ concentration needed for the physiologically important accumulation of $\mathrm{Ca}^{2+}$ is reduced when the dielectric coefficient of the protein is reduced; the affinity of the channel for $\mathrm{Ca}^{2+}$ increases. As a measure of the $\mathrm{Ca}^{2+} / \mathrm{Na}^{+}$selectivity in this accumulation, we consider the bath $\mathrm{CaCl}_{2}$ concentration at which equal numbers of $\mathrm{Ca}^{2+}$ and $\mathrm{Na}^{+}$are accumulated in the pore cylinder $(\Delta H=10 \AA)$ and the central region of the pore cylinder $(\Delta H=4.2 \AA)$.

This characteristic bath concentration of $\mathrm{Ca}^{2+}$ is reduced when the dielectric coefficient is varied from 80 to smaller values and passes through a minimum (indicating maximal selectivity) whose position depends on the size of the pore region where accumulation is assessed (Fig. 9). By this measure, $\mathrm{Ca}^{2+}$ selectivity in the central section of the model pore $(\Delta H=4.2 \AA)$ can be increased by about an order of magnitude (from 400 to $60 \mu \mathrm{M}$ ) by reducing the dielectric coefficient of the protein. Calculating occupancy in both the center of the pore and in the entire pore, the reduction of selectivity occurring at small dielectric coefficients reflects saturation of $\mathrm{Ca}^{2+}$ occupancy. To balance the increasing amount of induced charge on the pore wall as $\epsilon$ is decreased below 10 , 


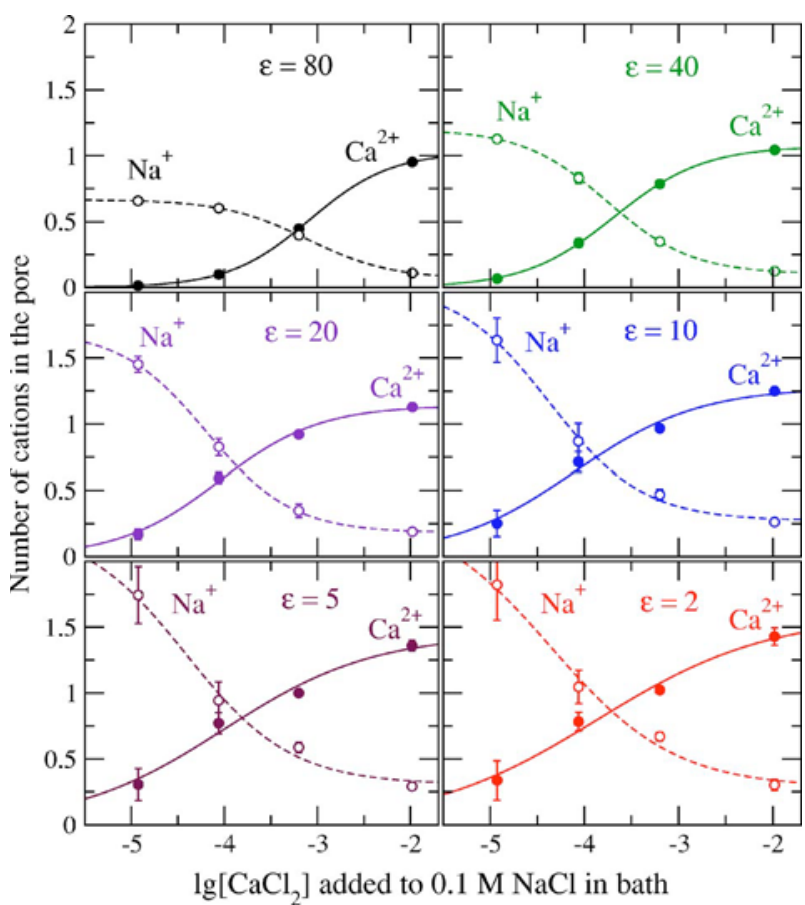

FIG. 8. (Color online) $\mathrm{Ca}^{2+}$ displaces $\mathrm{Na}^{+}$from the pore as $\left[\mathrm{CaCl}_{2}\right]$ increases. The average numbers of $\mathrm{Na}^{+}$and $\mathrm{Ca}^{2+}$ ions with centers in the cylindrical pore regions $(\Delta H=10 \AA)$ are plotted vs the logarithm of $\mathrm{CaCl}_{2}$ concentration in the bath; the bath concentration of $\mathrm{NaCl}$ is maintained at $0.1 M$. The symbols with error bars are simulation results; curves [based on Eq. (8) of Ref. 20] are fitted to aid the eye; the fit includes a point obtained in the absence of $\mathrm{CaCl}_{2}$ (not shown).

more $\mathrm{Na}^{+}$is absorbed in the channel producing the turnover in selectivity when $\epsilon<20$. This additional $\mathrm{Na}^{+}$accumulates chiefly near the pore entrances. As a result, the occupancy curves using the value $\Delta H=4.2 \AA$ (Fig. 4 of supplementary material ${ }^{37}$ ) show greater selectivity with a much less pronounced minimum (Fig. 9, open circles). The minimum in the $\left[\mathrm{CaCl}_{2}\right]$ vs $\epsilon$ curve indicates that $\mathrm{Ca}^{2+}$ affinity can be adjusted to a maximal value under these conditions, but the generality of this effect of dielectric coefficient needs to be studied further.

The presence of the dielectric boundary thus has marked effects on the amounts of $\mathrm{Ca}^{2+}$ and $\mathrm{Na}^{+}$accumulated in the pore as well as the net ionic charge accumulated in the pore $\left[Q_{\text {net }}\right.$, see Fig. 10(a) $]$. Because the induced charge attracts

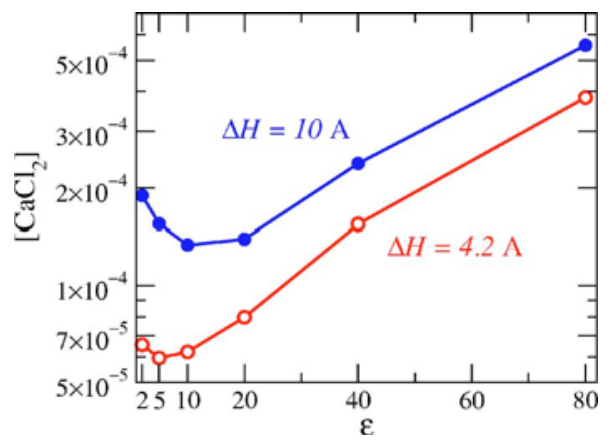

FIG. 9. (Color online) The dielectric coefficient of the protein controls the $\mathrm{Ca}^{2+} / \mathrm{Na}^{+}$selectivity of the model pore. We assess selectivity using the bath $\mathrm{CaCl}_{2}$ concentration from which the pore cylinder (or central region of the pore cylinder) absorbs equal numbers of $\mathrm{Ca}^{2+}$ and $\mathrm{Na}^{+}$.

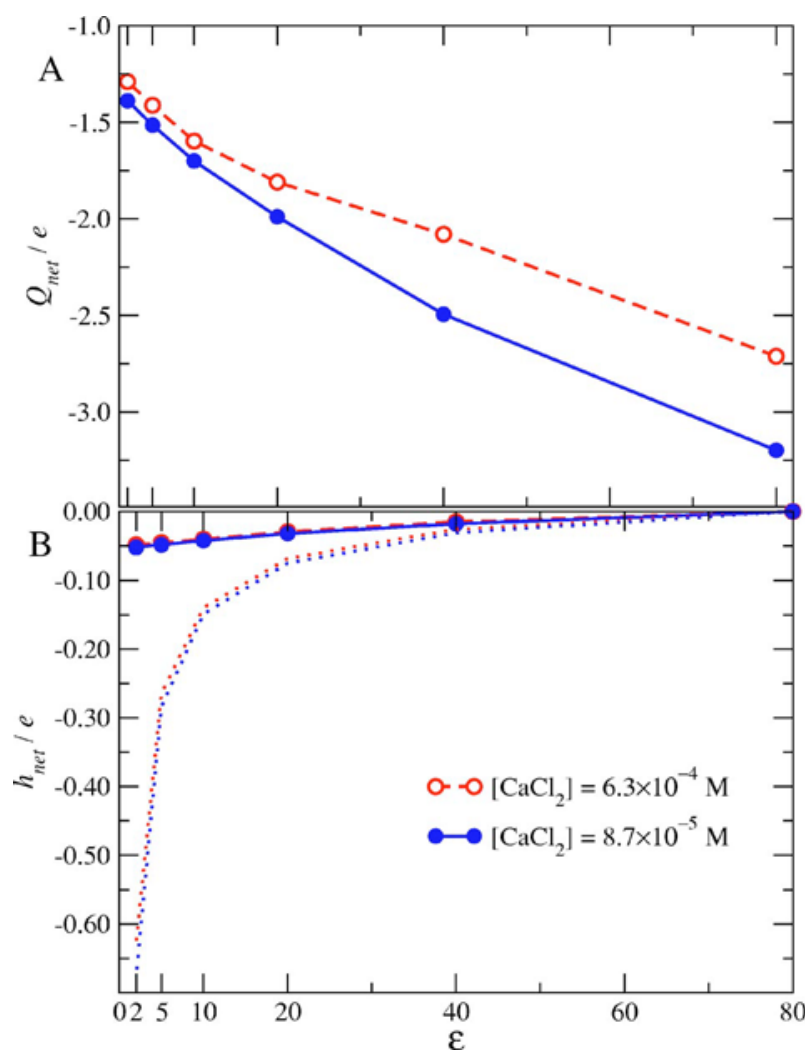

FIG. 10. (Color online) Comparing ionic net charge in the pore and charge induced on the pore wall. (A) Ionic net charge $\left(Q_{\text {net }}\right)$ in the cylindrical part of the pore. (B) Total charge induced on the cylindrical wall of the pore $\left(h_{\text {net }}\right)$. The dotted curves of the bottom panel represent $h_{\text {net }}^{\text {closed }}=Q_{\text {net }}(1 / \epsilon$ $-1 / 80)$, the charge that would be induced by $Q_{\text {net }}$ on the wall of a closed cavity lined by the protein.

cations into the filter, we also plot the net induced charge $h_{\text {net }}$ on the wall of the cylindrical pore region $(|z| \leqslant 5 \AA)$ in Fig. 10(b). A small dielectric coefficient forces the ionic balance in the pore toward local electroneutrality [Fig. 10(a)] as more counterions are attracted (see also Fig. 8). Nevertheless, local electroneutrality is never achieved, even when the dielectric coefficient approaches $\epsilon=2$. The induced charge $h_{\text {net }}$ is always negative [Fig. 10(b)], as is the net ionic charge in the pore $Q_{\text {net }}[$ Fig. 10(a)].

The induced charge is surprisingly small, given the magnitude of $Q_{\text {net }}$ in the pore. To illustrate this, consider any closed surface that includes the net charge $Q_{\text {net }}$. If the dielectric cofficient of the inside is 80 and the dielectric cofficient of the outside is $\epsilon$, then the total charge induced on the closed surface is $h_{\text {net }}^{\text {closed }}=Q_{\text {net }}(1 / \epsilon-1 / 80)$ [plotted in Fig. 10(b) as dotted lines]. ${ }^{61}$ The small size of the induced charge $h_{\text {net }}$ in the pore geometry compared to $h_{\text {net }}^{\text {closed indicates that }}$ the electric field from the net charge in the pore is largely restricted to the aqueous region of the system and does not cross the pore wall. The net ionic charge is screened by interactions occurring through the mouths of the pore. Indeed, a cloud of chiefly $\mathrm{Na}^{+}$cations dwells just outside the narrow part of the pore and forms a double layer with the net charge in the pore (Fig. 5). This nonlocal neutralization of the ionic net charge appears to be sufficient to almost abolish the electric field reaching from the pore into the weak dielectric of the protein, and hence to almost abolish the induction of 


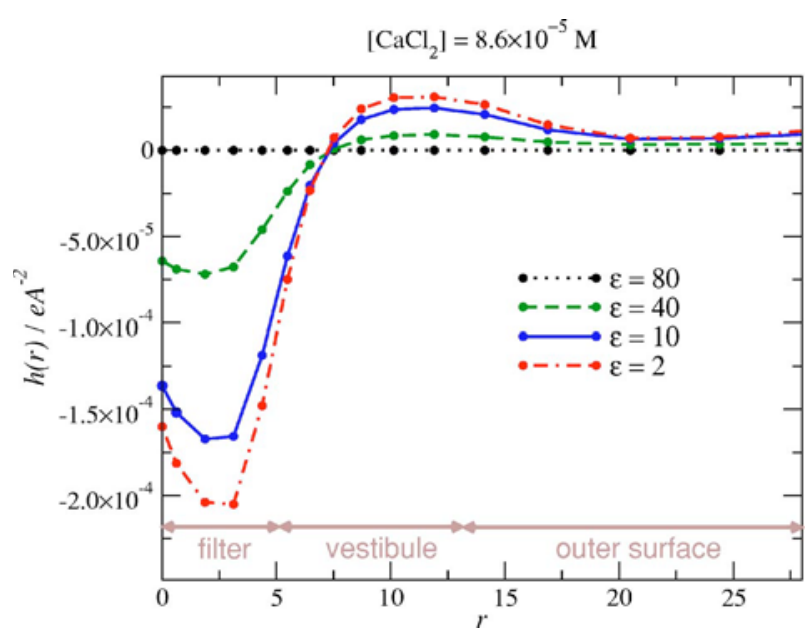

FIG. 11. (Color online) Distribution of induced charge on the protein surface. The plotted induced charge densities apply to loci along a surface path from point A to point B in Fig. 1(a) and are averaged over the angle of revolution and the two faces of the protein.

charge at that boundary. The distribution of the induced charge is shown in Fig. 11. The polarity of the induced charge indicates that the electric field crossing the boundary reverses polarity in the vestibular region near the pore as required by Gauss's law that the total induced charge on the protein surface is zero (see the sum rule discussed in Sec. III B 3).

Although the induced charge itself is small, the presence of the boundary with a weaker dielectric has a profound effect: the number of cationic counterions attracted into the pore substantially increases as the dielectric coefficient of the protein is reduced (Fig. 7). The local balance of ionic charge in the pore is thereby improved, and the field spreading from the pore into the protein is reduced. The small magnitude of the induced charge is the result of a strong negative feedback: the electrostatic energy of the system would substantially increase if the ions in the pore created a significant electrical field across the weak dielectric of the protein.

The absorption of more ions into the pore is of potential physiological importance, because it allows the pore to use excluded volume to distinguish ions by size and thus to develop a selectivity for cations with larger valence or smaller size (CSC mechanism ${ }^{16-18,20,21}$ ). Biological $\mathrm{Ca}$ channels have this kind of selectivity. Our simulations have used a pore model of rather large radius ( $4.5 \AA)$. More narrow pore geometries are possible and might yield stronger selectivity. Simulations exploring this possibility will be presented in a later paper.

The analysis here uses a low-resolution representation of the dielectric properties of the protein and ionic solution. But it is important to remember that the physical phenomena described this way actually occur and must be visible and accurately computable if higher resolution simulations are to be more reliable than reduced models. In particular, induced charge will appear at any boundary separating materials in which electric fields move matter differently. High-resolution simulations must resolve such effects and compute them with calibrated accuracy as they extend and eventually replace the low-resolution methods used here.

\section{SUMMARY}

We describe a method to include dielectrics of complex geometry into MC simulations of charged systems. The accuracy of the method is verified, and the method is shown to be efficient for calculating the system's electrostatic energy in every step of a simulation. The method is used to simulate a reduced model of ionic selectivity in biological $\mathrm{Ca}$ channels.

Early theoretical studies of channels found that ions cannot cross an ion channel if the protein has a low dielectric coefficient because the ions induce repulsive surface charges on the dielectric interface. ${ }^{31-35}$ Here we find that the same physics can increase the absorption of cations into the pore (compared to having no dielectric interfaces) if the channel pore contains negative charges from the protein; with a low protein dielectric coefficient, the negative protein charges induce negative surface charges on the dielectric interface that attract cations. Moreover, $\mathrm{Ca}^{2+}$ is preferentially absorbed over $\mathrm{Na}^{+}$, indicating the possibility that $\mathrm{Ca}$ channels use their dielectric properties to regulate their affinity for $\mathrm{Ca}^{2+}$.

\section{ACKNOWLEDGMENTS}

The authors are grateful for the support of the Hungarian National Research Fund [OTKA K63322, to the authors (D.B. and M.V.)], NATO Grant No. PST.CLG.980366 [to the authors (D.H., D.G., and D.B.)], and NIH Grant No. GM067241 [to the authors (B.E. and D.G.)]. The use of the facilities of the Ira and Marylou Fulton Supercomputing Center at BYU is acknowledged with thanks.

${ }^{1}$ J. S. Smith, T. Imagawa, J. Ma, M. Fill, K. P. Campbell, and R. Coronado, J. Gen. Physiol. 92, 1 (1988).

${ }^{2}$ W. A. Sather and E. W. McCleskey, Annu. Rev. Physiol. 65, 133 (2003).

${ }^{3}$ D. Gillespie, L. Xu, Y. Wang, and G. Meissner, J. Phys. Chem. B 109, 15598 (2005).

${ }_{5}^{4} \mathrm{M}$. Fill (private communication).

${ }^{5}$ S. H. Heinemann, H. Terlau, W. Stuhmer, K. Imoto, and S. Numa, Nature (London) 356, 441 (1992).

${ }^{6}$ J. Yang, P. T. Ellinor, W. A. Sather, J. F. Zhang, and R. W. Tsien, Nature (London) 366, 158 (1993).

${ }^{7}$ P. T. Ellinor, J. Yang, W. A. Sather, J. F. Zhang, and R. W. Tsien, Neuron 15, 1121 (1995).

${ }^{8}$ L. Gao, D. Balshaw, L. Xu, A. Tripathy, C. Xin, and G. Meissner, Biophys. J. 79, 828 (2000).

${ }^{9}$ Y. Wang, L. Xu, D. A. Pasek, D. Gillespie, and G. Meissner, Biophys. J. 89, 256 (2005).

${ }^{10}$ L. Xu, Y. Wang, D. Gillespie, and G. Meissner, Biophys. J. 90, 443 (2006).

${ }^{11}$ C. K. Schauer and P. P. Anderson, J. Am. Chem. Soc. 109, 3646 (1987).

${ }^{12}$ M. R. Nelson and W. J. Chazin, BioMetals 11, 297 (1998).

${ }^{13} \mathrm{http}: / /$ structbio.vanderbilt.edu/cabp_database/cabp.html

${ }^{14}$ B. Corry and S.-H. Chung, Cell. Mol. Life Sci. 63, 301 (2006).

${ }^{15}$ B. Corry, T. Vora, and S.-H. Chung, Biochim. Biophys. Acta 1711, 72 (2005).

${ }^{16}$ D. Boda, D. Henderson, and D. D. Busath, Mol. Phys. 100, 2361 (2002).

${ }^{17}$ D. Boda, D. Henderson, and D. D. Busath, J. Phys. Chem. B 105, 11574 (2001).

${ }^{18}$ W. Nonner, D. Gillespie, D. Henderson, and B. Eisenberg, J. Phys. Chem. B 105, 6427 (2001).

${ }^{19}$ B. Corry, T. Allen, S. Kuyucak, and S.-H. Chung, Biophys. J. 80, 195 (2001).

${ }^{20}$ D. Boda, D. D. Busath, D. Henderson, and S. Sokołowski, J. Phys. Chem. B 104, 8903 (2000).

${ }^{21}$ W. Nonner, L. Catacuzzeno, and B. Eisenberg, Biophys. J. 79, 1976 (2000). 
${ }^{22}$ W. Nonner and B. Eisenberg, Biophys. J. 75, 1287 (1998).

${ }^{23}$ M. Hoyles, S. Kuyucak, and S.-H. Chung, Phys. Rev. E 58, 3654 (1998)

${ }^{24}$ S.-H. Chung, M. Hoyles, T. Allen, and S. Kuyucak, Biophys. J. 75, 793 (1998).

${ }^{25}$ R. Roth and D. Gillespie, Phys. Rev. Lett. 95, 054706 (2005).

${ }^{26}$ H. Miedema, A. Meter-Arkema, J. Wierenga, J. Tang, B. Eisenberg, W. Nonner, H. Hektor, D. Gillespie, and W. Meijberg, Biophys. J. 87, 3137 (2004).

${ }^{27}$ M. Vrouenraets, J. Wierenga, W. Meijberg, and H. Miedema, Biophys. J. 90, 2102 (2006).

${ }^{28}$ Z. S. Siwy, M. R. Powell, E. Kalman, R. D. Astumian, and R. S. Eisenberg, Nano Lett. 6, 473 (2006).

${ }^{29}$ D. Gillespie, W. Nonner, and R. S. Eisenberg, J. Phys.: Condens. Matter 14, 12129 (2002)

${ }^{30}$ D. Gillespie, W. Nonner, and R. S. Eisenberg, Phys. Rev. E 68, 031503 (2003).

${ }^{31}$ V. A. Parsegian, Nature (London) 221, 844 (1969).

${ }^{32}$ B. Neumcke and P. Läuger, Biophys. J. 9, 1160 (1969).

${ }^{33}$ D. G. Levitt, Biophys. J. 22, 209 (1978).

${ }^{34}$ D. G. Levitt, Biophys. J. 22, 221 (1978).

${ }^{35}$ P. C. Jordan, Biophys. J. 39, 157 (1982).

${ }^{36}$ D. Boda, D. Gillespie, W. Nonner, D. Henderson, and B. Eisenberg, Phys. Rev. E 69, 046702 (2004).

${ }^{37}$ See EPAPS Document No. E-JCPSA6-125-514625 for description of surface disretization and figures on control simulations. This document can be reached via a direct link in the online article's HTML reference section or via the EPAPS homepage (http://www.aip.org/pubservs/epaps.html).

${ }^{38}$ M. P. Allen and D. J. Tildesley, Computer Simulation of Liquids (Oxford, New York, 1987).

${ }^{39}$ D. Frenkel and B. Smit, Understanding Molecular Simulations (Academic, San Diego, 1996).

${ }^{40}$ R. J. Sadus, Molecular Simulation of Fluids; Theory, Algorithms, and Object-Orientation (Elsevier, Amsterdam, 1999).

${ }^{41}$ J. P. Valleau and L. K. Cohen, J. Chem. Phys. 72, 5935 (1980).

${ }^{42}$ D. Boda, D. Gillespie, B. Eisenberg, W. Nonner, and D. Henderson, in Ionic Soft Matter: Novel Trends in Theory and Applications, NATO Science Series: II: Mathematics, Physics and Chemistry, edited by D. Hend- erson, M. Holovko, and A. Trokhymchuk (Springer, New York, 2005), Vol. 206.

${ }^{43}$ R. Allen, J.-P. Hansen, and S. Melchionna, Phys. Chem. Chem. Phys. 3, 4117 (2001).

${ }^{44}$ R. Allen, S. Melchionna, and J.-P. Hansen, Phys. Rev. Lett. 89, 175502 (2002).

${ }^{45}$ R. Allen, S. Melchionna, and J.-P. Hansen, J. Phys.: Condens. Matter 15, S297 (2003).

${ }^{46}$ R. Allen, J.-P. Hansen, and S. Melchionna, J. Chem. Phys. 119, 3905 (2003).

${ }^{47}$ S. Miertus, E. Scrocco, and J. Tomasi, Chem. Phys. 55, 117 (1981).

${ }^{48}$ J. Tomasi and M. Persico, Chem. Rev. (Washington, D.C.) 94, 2027 (1994).

${ }^{49}$ A. Klamt and G. Schüürmann, J. Chem. Soc., Perkin Trans. 1 2, 799 (1993).

${ }^{50}$ B. Mennucci, E. Cancès, and J. Tomasi, J. Phys. Chem. B 101, 10506 (1997).

${ }^{51}$ B. Mennucci and E. Cancès, J. Math. Chem. 23, 309 (1998).

${ }^{52}$ B. Mennucci, R. Cammi, and J. Tomasi, J. Chem. Phys. 109, 2798 (1998).

${ }^{53}$ H. Hoshi, M. Sakurai, Y. Inoue, and R. Chûjô, J. Chem. Phys. 87, 1107 (1987).

${ }^{54}$ R. Cammi and J. Tomasi, J. Chem. Phys. 100, 7495 (1994).

${ }^{55}$ R. Cammi and J. Tomasi, J. Chem. Phys. 101, 3888 (1994).

${ }^{56}$ C. S. Pomelli, J. Tomasi, and V. Barone, Theor. Chem. Acc. 105, 446 (2001).

${ }^{57}$ L. Frediani, R. Cammi, S. Corni, and J. Tomasi, J. Chem. Phys. 120, 3893 (2004)

${ }^{58}$ M. E. Green and J. Lu, Prog. Colloid Polym. Sci. 171, 117 (1997).

${ }^{59}$ M. E. Green and J. Lu, J. Phys. Chem. B 101, 6512 (1997).

${ }^{60}$ J. Lu and M. E. Green, J. Phys. Chem. B 103, 2776 (1999).

${ }^{61}$ D. J. Griffiths, Introduction to Electrodynamics (Prentice-Hall, Englewood Cliffs, 1999).

${ }^{62}$ M. Hoyles, S. Kuyucak, and S.-H. Chung, Comput. Phys. Commun. 115, 45 (1998).

${ }^{63}$ F. Chen and D. M. Chipman, J. Chem. Phys. 119, 10289 (2003).

${ }^{64}$ D. M. Chipman and M. Dupuis, Theor. Chem. Acc. 107, 90 (2002). 